\title{
A New Sum Analogous to Gauss Sums and Its Fourth Power Mean
}

\author{
Shaofeng Ru${ }^{1}$ and Wenpeng Zhang ${ }^{2}$ \\ ${ }^{1}$ School of Economics \& Management, Northwest University, Xian 710127, China \\ ${ }^{2}$ Department of Mathematics, Northwest University, Xian 710127, China \\ Correspondence should be addressed to Wenpeng Zhang; wpzhang@nwu.edu.cn
}

Received 19 January 2014; Accepted 12 May 2014; Published 21 May 2014

Academic Editor: Paolo Vannucci

Copyright (C) 2014 S. Ru and W. Zhang. This is an open access article distributed under the Creative Commons Attribution License, which permits unrestricted use, distribution, and reproduction in any medium, provided the original work is properly cited.

The main purpose of this paper is to use the analytic methods and the properties of Gauss sums to study the computational problem of one kind of new sum analogous to Gauss sums and give an interesting fourth power mean and a sharp upper bound estimate for

\section{Introduction}

Let $q \geq 3$ be an integer, and let $\chi$ be a Dirichlet character $\bmod q$. Then for any integer $n$, famous Gauss sum $G(\chi, n)$ is defined as follows:

$$
G(\chi, n)=\sum_{a=1}^{q} \chi(a) \cdot e\left(\frac{n a}{q}\right),
$$

where $e(y)=e^{2 \pi i y}$.

This sum plays a very important role in the study of analytic number theory; many famous number theoretic problems are closely related to it. For example, the distribution of primes, Goldbach problem, the estimate of character sums, and the properties of Dirichlet $L$-functions are some good examples.

It is clear that if $\chi$ is a primitive Dirichlet character $\bmod q$, then we have $G(\chi, n)=\bar{\chi}(n) \cdot G(\chi, 1) \equiv \bar{\chi}(n) \cdot \tau(\chi)$ and $|\tau(\chi)|=$ $\sqrt{q}$. Many properties of $G(\chi, n)$ and $\tau(\chi)$ can be found in [13].

In this paper, we introduce a new sum analogous to Gauss sums as follows:

$$
G(\chi, b, c, m ; q)=\sum_{a=0}^{q-1} \chi\left(a^{2}+b a+c\right) \cdot e\left(\frac{m a}{q}\right),
$$

where $\chi$ is a character $\bmod q$, and $b, c$, and $m$ are any integers with $(m, q)=1$.
The main purpose of this paper is to study the properties of $G(\chi, b, c, m ; q)$, such as the following two problems:

(A) giving an upper bound estimate of $G(\chi, b, c, m ; q)$;

(B) the problem of whether there exists a computational formula for the $2 k$ th power mean

$$
\sum_{c=0}^{q-1}\left|\sum_{a=0}^{q-1} \chi\left(a^{2}+b a+c\right) \cdot e\left(\frac{m a}{q}\right)\right|^{2 k}, \quad k \geq 2 .
$$

It seems that no one has studied these problems yet; at least we have not seen any related results in the existing literature. The problems are interesting, because there exists a close relationship between the sum $G(\chi, b, c, m ; q)$ and the generalized Kloosterman sums; they can also help us to further understand the properties of hybrid mean value of an exponential sum and character of a polynomial.

For general integer $q>3$, these two problems seem to be hard to make progress. But if $q=p>2$ is a prime and $k=2$, then we can prove the following two conclusions.

Theorem 1. Let $p$ be an odd prime and $\chi$ any nonprincipal character $\bmod p$. Then for any integers $b, c$, and $m$ with $(m, p)=1$, one has the estimate

$$
\left|\sum_{a=0}^{p-1} \chi\left(a^{2}+b a+c\right) \cdot e\left(\frac{m a}{p}\right)\right| \leq 2 \sqrt{p} .
$$


Theorem 2. Let $p$ be an odd prime and $\chi$ any nonprincipal character $\bmod p$. Then for any integers $b$ and $m$ with $(m, p)=$ 1 , one has the identity

$$
\begin{aligned}
& \sum_{c=0}^{p-1}\left|\sum_{a=0}^{p-1} \chi\left(a^{2}+b a+c\right) \cdot e\left(\frac{m a}{p}\right)\right|^{4} \\
& = \begin{cases}2 p^{3}-3 p^{2}-3 p, & \text { if } \chi \text { is the Legendre symbol mod } p \\
2 p^{3}-6 p^{2}, & \text { if } \chi \text { is a nonreal character } \bmod p .\end{cases}
\end{aligned}
$$

Some Notes. If $\chi=\chi_{0}$ is the principal character $\bmod p$, then note that $\chi_{0}\left(a^{2}+b a+c\right)=0$ or 1 and $\left|G\left(\chi_{0}, b, c, m ; p\right)\right| \leq 3$. So, in this case, the result is trivial; we do not need to discuss problems (A) and (B).

For any integer $k \geq 3$, whether there exists an exact computational formula for the $2 k$ th power mean

$$
\sum_{c=0}^{p-1}\left|\sum_{a=0}^{p-1} \chi\left(a^{2}+b a+c\right) \cdot e\left(\frac{m a}{p}\right)\right|^{2 k}
$$

is an open problem.

Furthermore, for general integer $q>3$, whether there exists a nontrivial upper bound estimate for $G(\chi, b, c, m ; q)$ is also an interesting problem.

\section{Several Lemmas}

In this section, we will give several lemmas, which are necessary in the proof of our theorems. Hereinafter, we will use many properties of character sums and Gauss sums; all of these can be found in $[1,4]$. So they will not be repeated here. First we have the following.

Lemma 3. Let $p$ be an odd prime; then, for any integer $c$ with $(c, p)=1$, one has the identity

$$
\sum_{a=0}^{p-1} e\left(\frac{c a^{2}}{p}\right)=\left(\frac{c}{p}\right) \cdot \tau\left(\chi_{2}\right)
$$

where $\chi_{2}=(* / p)$ denotes the Legendre symbol.

Proof. From the properties of Gauss sums and quadratic residue $\bmod p$ we have

$$
\begin{aligned}
\sum_{a=0}^{p-1} e\left(\frac{c a^{2}}{p}\right) & =1+\sum_{a=1}^{p-1} e\left(\frac{c a^{2}}{p}\right) \\
& =1+\sum_{a=1}^{p-1}\left(1+\left(\frac{a}{p}\right)\right) \cdot e\left(\frac{c a}{p}\right) \\
& =\sum_{a=0}^{p-1} e\left(\frac{c a}{p}\right)+\sum_{a=1}^{p-1}\left(\frac{a}{p}\right) \cdot e\left(\frac{c a}{p}\right) \\
& =\left(\frac{c}{p}\right) \sum_{a=1}^{p-1}\left(\frac{a}{p}\right) \cdot e\left(\frac{a}{p}\right)=\left(\frac{c}{p}\right) \cdot \tau\left(\chi_{2}\right) .
\end{aligned}
$$

This proves Lemma 3.
Lemma 4. Let $p$ be an odd prime and $\chi$ any nonprincipal Dirichlet character mod $p$. Then for any integers $b$ and $c$, one has the identity

$$
\begin{aligned}
& \left|\sum_{a=0}^{p-1} \chi\left(a^{2}+b a+c\right) \cdot e\left(\frac{a}{p}\right)\right| \\
& \quad=\left|\sum_{r=1}^{p-1} \bar{\chi} \chi_{2}(r) \cdot e\left(\frac{\left(4 c-b^{2}\right) r-\overline{16} \cdot \bar{r}}{p}\right)\right|,
\end{aligned}
$$

where $\bar{r}$ denotes the solution of the congruence equation $r \cdot x \equiv$ $1 \bmod p$.

Proof. Since $\chi$ is a nonprincipal Dirichlet character $\bmod p$, from Lemma 3 and the properties of Gauss sums we have

$$
\begin{aligned}
& \sum_{a=0}^{p-1} \chi\left(a^{2}+b a+c\right) \cdot e\left(\frac{a}{p}\right) \\
& =\frac{1}{\tau(\bar{\chi})} \cdot \sum_{a=0}^{p-1} \sum_{r=1}^{p-1} \bar{\chi}(r) e\left(\frac{r\left(a^{2}+b a+c\right)}{p}\right) \cdot e\left(\frac{a}{p}\right) \\
& =\frac{1}{\tau(\bar{\chi})} \cdot \sum_{r=1}^{p-1} \bar{\chi}(r) \\
& \times \sum_{a=0}^{p-1} e\left(\frac{r a^{2}+(b r+1) a+c r}{p}\right) \\
& =\frac{1}{\tau(\bar{\chi})} \cdot \sum_{r=1}^{p-1} \bar{\chi}(r) \\
& \times \sum_{a=0}^{p-1} e\left(\frac{\overline{4} r(2 a+\bar{r}(b r+1))^{2}+c r-\overline{4} \bar{r}(b r+1)^{2}}{p}\right) \\
& =\frac{1}{\tau(\bar{\chi})} \cdot \sum_{r=1}^{p-1} \bar{\chi}(r) \\
& \times \sum_{u=0}^{p-1} e\left(\frac{\overline{4} r u^{2}+\overline{4} \cdot\left(4 c-b^{2}\right) r-\overline{2} \cdot b-\overline{4} \cdot \bar{r}}{p}\right) \\
& =\frac{1}{\tau(\bar{\chi})} \cdot \sum_{r=1}^{p-1} \bar{\chi}(r)\left(\frac{\overline{4} \cdot r}{p}\right) \cdot \tau\left(\chi_{2}\right) \\
& e\left(\frac{\overline{4} \cdot\left(4 c-b^{2}\right) r-\overline{2} \cdot b-\overline{4} \cdot \bar{r}}{p}\right) \\
& =\frac{\tau\left(\chi_{2}\right)}{\tau(\bar{\chi})} \cdot \bar{\chi}(4) \cdot e\left(\frac{-\overline{2} \cdot b}{p}\right) \cdot \sum_{r=1}^{p-1} \bar{\chi}(r)\left(\frac{r}{p}\right) \\
& e\left(\frac{\left(4 c-b^{2}\right) r-\overline{16} \cdot \bar{r}}{p}\right) \text {. }
\end{aligned}
$$


For any nonprincipal character $\chi \bmod p$, we have $|\tau(\chi)|=$ $\sqrt{p}$. So, from $(10)$ and noting that $|\bar{\chi}(4)|=|e(-\overline{2} \cdot b / p)|=1$, we have

$$
\begin{aligned}
& \left|\sum_{a=0}^{p-1} \chi\left(a^{2}+b a+c\right) \cdot e\left(\frac{a}{p}\right)\right| \\
& \quad=\left|\sum_{r=1}^{p-1} \bar{\chi} \chi_{2}(r) \cdot e\left(\frac{\left(4 c-b^{2}\right) r-\overline{16} \cdot \bar{r}}{p}\right)\right| .
\end{aligned}
$$

This proves Lemma 4 .

Lemma 5. Let $p$ be an odd prime and $\chi$ any Dirichlet character $\bmod p$. Then for any integers $m$ and $n$, one has the estimate

$$
\sum_{a=1}^{p-1} \chi(a) \cdot e\left(\frac{m a+n \bar{a}}{p}\right) \leq 2 \sqrt{p} \cdot(m, n, p)^{1 / 2}
$$

where $(m, n, p)$ denotes the greatest common divisor of $m, n$, and $p$.

Proof. This estimate is by Weil [5], Chowla [6], Malyshev [7], and Estermann [8] with some minor modifications.

Lemma 6. Let $p$ be an odd prime; then, for any integer $n$ with $(n, p)=1$, one has the calculating formula

$$
\begin{aligned}
& \sum_{m=1}^{p-1}\left|\sum_{a=1}^{p-1} \chi(a) \cdot e\left(\frac{m a+n \bar{a}}{p}\right)\right|^{4} \\
& =\left\{\begin{array}{cl}
2 p^{3}-3 p^{2} \\
-3 p-1, & \text { if } \chi \text { is the principal character } \bmod p ; \\
3 p^{3}-8 p^{2}, & \text { if } \chi \text { is the Legendre symbol } \bmod p ; \\
p^{2}(2 p-7), & \text { if } \chi \text { is a nonreal character } \bmod p .
\end{array}\right.
\end{aligned}
$$

Proof. See [9] or Corollary 2 of [10].

\section{Proof of the Theorems}

In this section, we will complete the proof of our theorems. First note that if $a$ passes through a reduced residue system $\bmod p$, then $m a$ also passes through a reduced residue system $\bmod p$, if $(m, p)=1$. From these properties we have

$$
\begin{aligned}
& \left|\sum_{a=0}^{p-1} \chi\left(a^{2}+b a+c\right) \cdot e\left(\frac{m a}{p}\right)\right| \\
& \quad=\left|\sum_{a=0}^{p-1} \chi\left(\bar{m}^{2} a^{2}+\bar{m} b a+c\right) \cdot e\left(\frac{a}{p}\right)\right| \\
& \quad=\left|\chi^{2}(\bar{m}) \sum_{a=0}^{p-1} \chi\left(a^{2}+m b a+c m^{2}\right) \cdot e\left(\frac{a}{p}\right)\right| \\
& \quad=\left|\sum_{a=0}^{p-1} \chi\left(a^{2}+m b a+c m^{2}\right) \cdot e\left(\frac{a}{p}\right)\right| .
\end{aligned}
$$

So without loss of generality we can assume that $m=1$. Now we prove Theorem 1. From Lemmas 4 and 5 we have

$$
\begin{aligned}
& \left|\sum_{a=0}^{p-1} \chi\left(a^{2}+b a+c\right) \cdot e\left(\frac{a}{p}\right)\right| \\
& \quad=\left|\sum_{r=1}^{p-1} \bar{\chi} \chi_{2}(r) \cdot e\left(\frac{\left(4 c-b^{2}\right) r-\overline{16} \cdot \bar{r}}{p}\right)\right| \\
& \quad \leq 2 \sqrt{p} \cdot\left(4 c-b^{2}, \overline{16}, p\right)^{1 / 2}=2 \sqrt{p} .
\end{aligned}
$$

This proves Theorem 1 .

Now we prove Theorem 2. From the properties of a complete residue system $\bmod p$ we know that if $c$ passes through a complete residue system $\bmod p$, then $4 c-b^{2}$ also passes through a complete residue system $\bmod p$. So from Lemma 4 we have

$$
\begin{aligned}
& \sum_{c=0}^{p-1}\left|\sum_{a=0}^{p-1} \chi\left(a^{2}+b a+c\right) \cdot e\left(\frac{a}{p}\right)\right|^{4} \\
& \quad=\sum_{c=0}^{p-1}\left|\sum_{r=1}^{p-1} \bar{\chi} \chi_{2}(r) \cdot e\left(\frac{\left(4 c-b^{2}\right) r-\overline{16} \cdot \bar{r}}{p}\right)\right|^{4} \\
& =\sum_{m=0}^{p-1}\left|\sum_{r=1}^{p-1} \bar{\chi} \chi_{2}(r) \cdot e\left(\frac{m r-\overline{16} \cdot \bar{r}}{p}\right)\right|^{4} \\
& =\sum_{m=0}^{p-1}\left|\sum_{r=1}^{p-1} \bar{\chi} \chi_{2}(r) \cdot e\left(\frac{m r+\bar{r}}{p}\right)\right|^{4} .
\end{aligned}
$$

If $\chi=\chi_{2}$ is the Legendre symbol, then $\chi \chi_{2}=\chi_{0}$ is the principal character mod $p$, so from (16) and Lemma 6 we have

$$
\begin{aligned}
& \sum_{c=0}^{p-1}\left|\sum_{a=0}^{p-1} \chi\left(a^{2}+b a+c\right) \cdot e\left(\frac{a}{p}\right)\right|^{4} \\
& \quad=\sum_{m=0}^{p-1}\left|\sum_{r=1}^{p-1} \bar{\chi} \chi_{2}(r) \cdot e\left(\frac{m r+\bar{r}}{p}\right)\right|^{4} \\
& =1+\sum_{m=1}^{p-1}\left|\sum_{r=1}^{p-1} e\left(\frac{m r+\bar{r}}{p}\right)\right|^{4} \\
& =1+2 p^{3}-3 p^{2}-3 p-1 \\
& =2 p^{3}-3 p^{2}-3 p .
\end{aligned}
$$


If $\chi$ is not a real character mod $p$, then from (16) and Lemma 6 we have

$$
\begin{aligned}
\sum_{c=0}^{p-1}\left|\sum_{a=0}^{p-1} \chi\left(a^{2}+b a+c\right) \cdot e\left(\frac{a}{p}\right)\right|^{4} \\
=\sum_{m=0}^{p-1}\left|\sum_{r=1}^{p-1} \bar{\chi} \chi_{2}(r) \cdot e\left(\frac{m r+\bar{r}}{p}\right)\right|^{4} \\
=\left|\sum_{r=1}^{p-1} \bar{\chi} \chi_{2}(r) \cdot e\left(\frac{\bar{r}}{p}\right)\right|^{4} \\
\quad+\sum_{m=1}^{p-1}\left|\sum_{r=1}^{p-1} \bar{\chi} \chi_{2}(r) \cdot e\left(\frac{m r+\bar{r}}{p}\right)\right|^{4} \\
=p^{2}+p^{2}(2 p-7)=2 p^{2}(p-3) .
\end{aligned}
$$

Now combining (14), (17), and (18) we may immediately deduce the identity

$$
\begin{aligned}
& \sum_{c=0}^{p-1}\left|\sum_{a=0}^{p-1} \chi\left(a^{2}+b a+c\right) \cdot e\left(\frac{m a}{p}\right)\right|^{4} \\
& = \begin{cases}2 p^{3}-3 p^{2}-3 p, & \text { if } \chi \text { is the Legendre symbol } \bmod p ; \\
2 p^{3}-6 p^{2}, & \text { if } \chi \text { is a nonreal character } \bmod p .\end{cases}
\end{aligned}
$$

This completes the proof of Theorem 2.

\section{Conflict of Interests}

The authors declare that there is no conflict of interests regarding the publication of this paper.

\section{Acknowledgments}

The authors would like to thank the referee for the very helpful and detailed comments, which have significantly improved the presentation of this paper. This work is supported by the P.S.F. (2013JZ001) and N.S.F. (11371291) of China.

\section{References}

[1] T. M. Apostol, Introduction to Analytic Number Theory, Springer, New York, NY, USA, 1976.

[2] K. Ireland and M. Rosen, A Classical Introduction to Modern Number Theory, Springer, New York, NY, USA, 1982.

[3] M. B. Nathanson, Additive Number Theory, the Classical Bases, vol. 164 of Graduate Texts in Mathematics, Springer, 1996.

[4] E. Alkan, "Values of Dirichlet $L$-functions, Gauss sums and trigonometric sums," Ramanujan Journal, vol. 26, no. 3, pp. 375398, 2011.

[5] A. Weil, "On some exponential sums," Proceedings of the National Academy of Sciences, vol. 34, pp. 204-207, 1949.

[6] S. Chowla, "On Kloosterman's Sum," Det Kongelige Norske Videnskabers Selskabs Forhandlinger, vol. 40, pp. 70-72, 1967.
[7] A. V. Malyshev, "A generalization of Kloosterman sums and their estimates," Vestnik Leningrad University, vol. 15, pp. 59-75, 1960 (Russian).

[8] T. Estermann, “On Kloostermann's sums," Mathematica, vol. 8, pp. 83-86, 1961.

[9] W. Zhang, "On the fourth power mean of the general Kloosterman sums," Indian Journal of Pure and Applied Mathematics, vol. 35, no. 2, pp. 237-242, 2004.

[10] L. Jianghua and L. Yanni, "Some new identities involving Gauss sums and general Kloosterman sums," Acta Mathematica Sinica, vol. 56, pp. 413-418, 2013 (Chinese). 


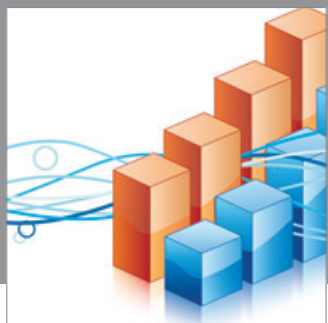

Advances in

Operations Research

mansans

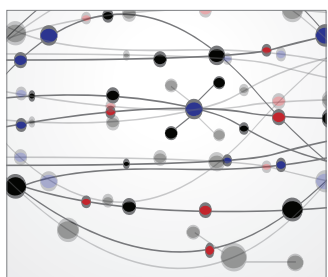

The Scientific World Journal
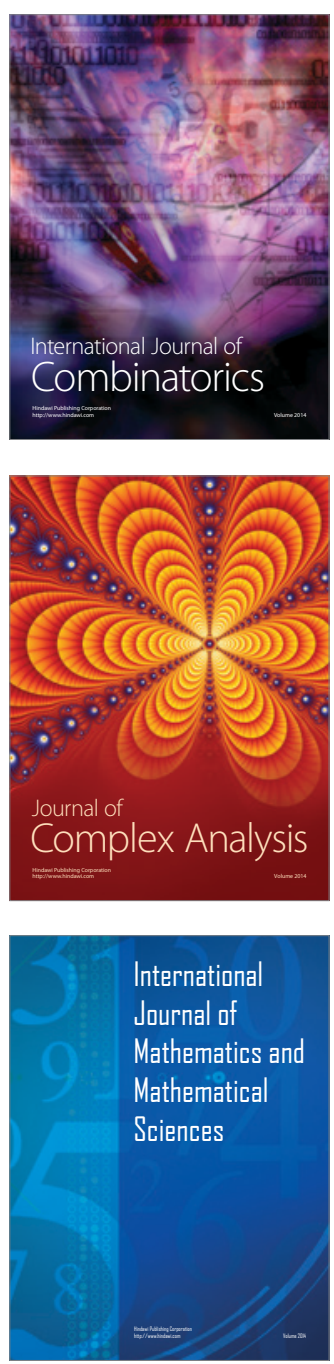
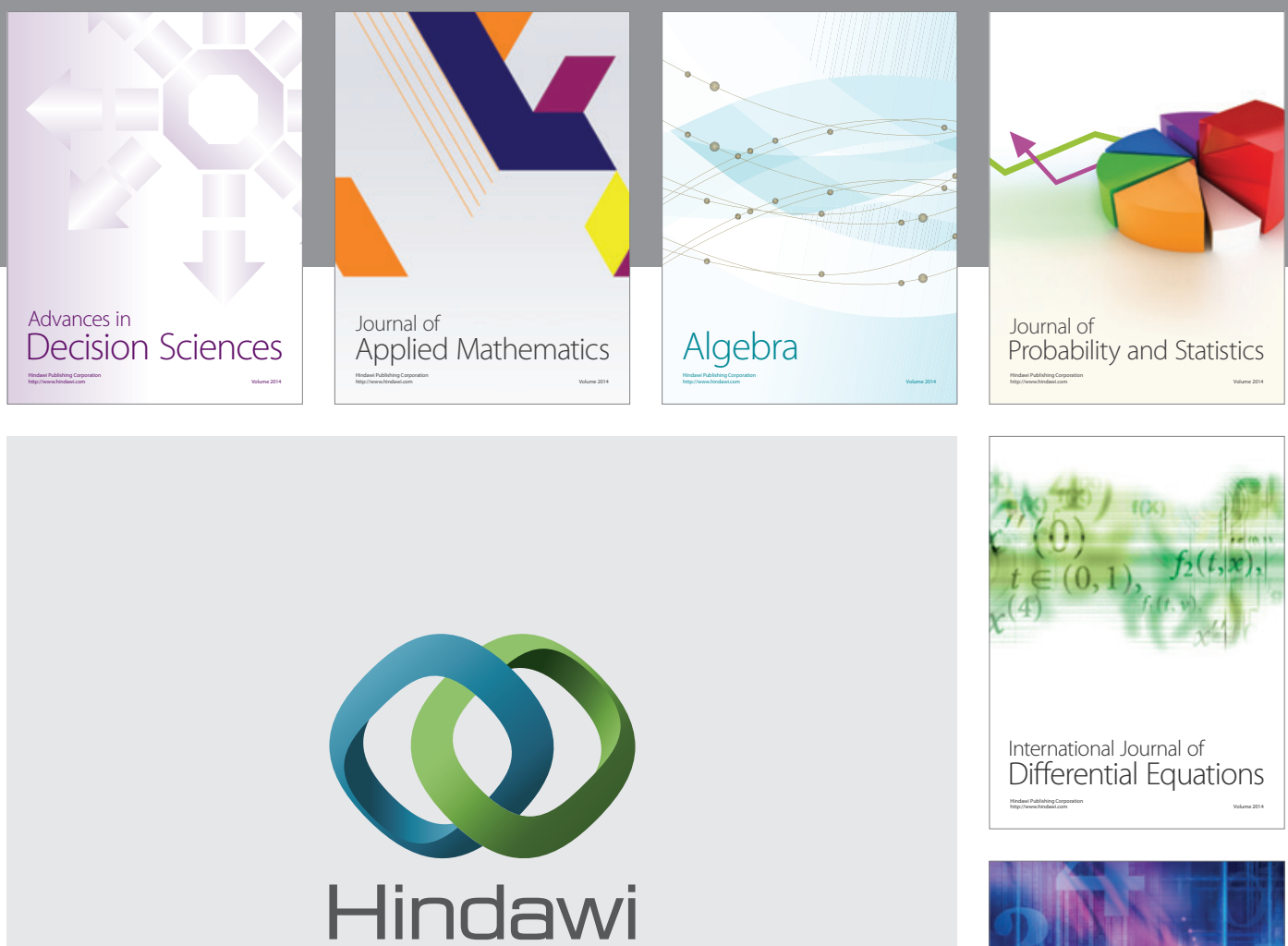

Submit your manuscripts at http://www.hindawi.com
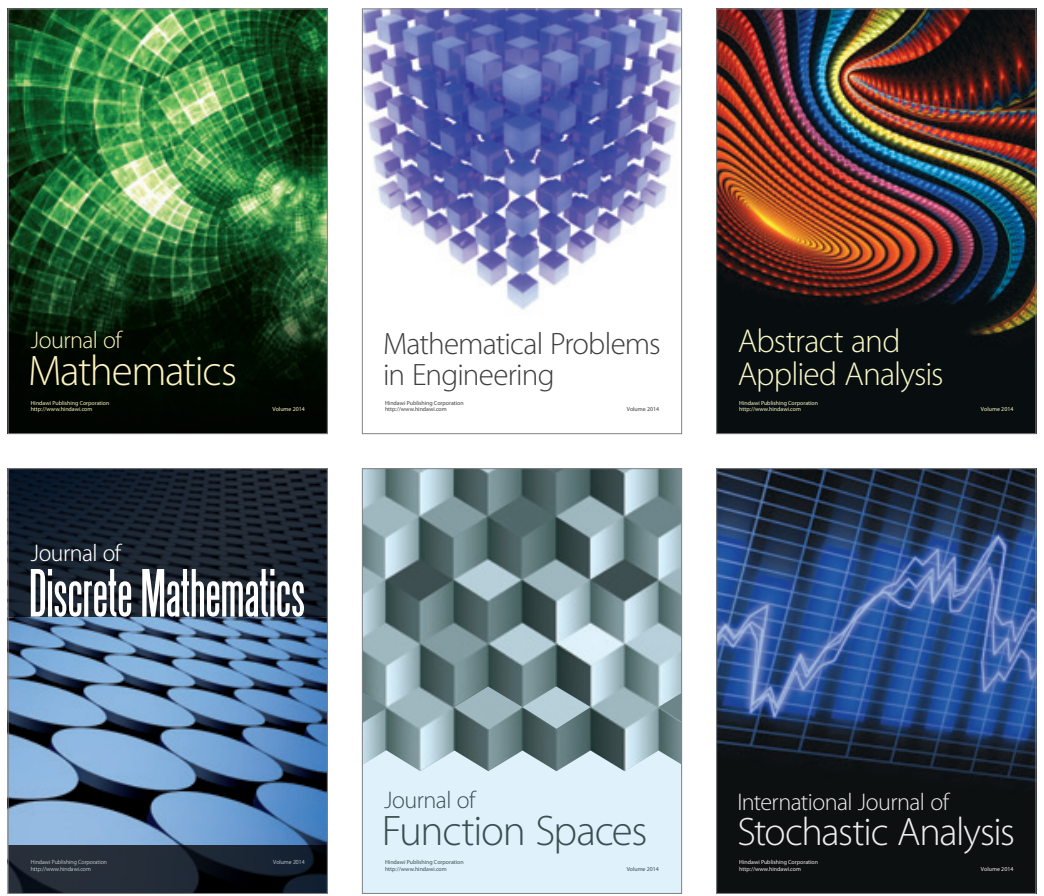

Journal of

Function Spaces

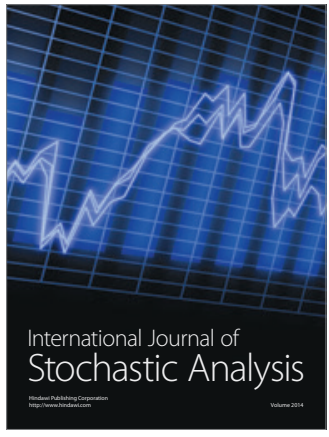

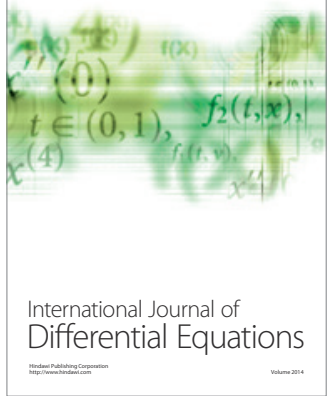
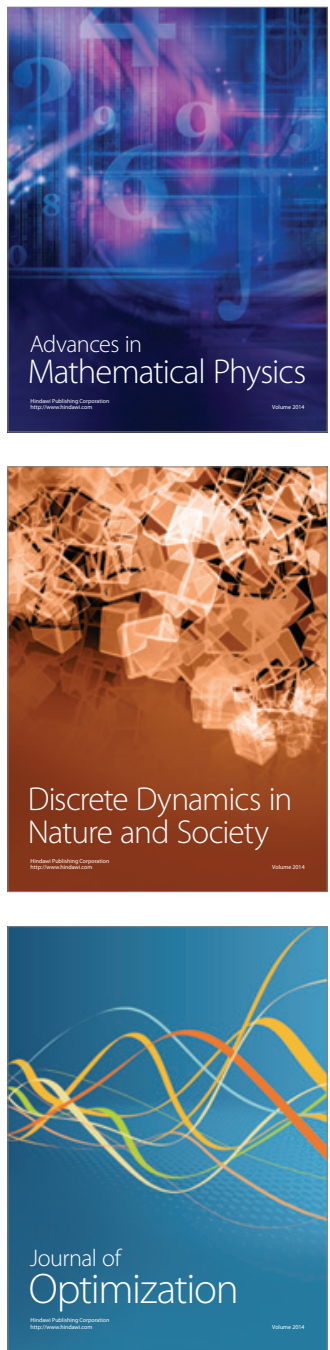